Nutzen und Risiko sorgfältig abwägen

\section{Bei Schwangeren nur dringende Impfungen vornehmen}

- Der Grundsatz der Ständigen Impfkommission (STIKO), nicht dringend indizierte Impfungen während der Schwangerschaft nicht vorzunehmen, gilt weiterhin, betonte Prof. Markus Knuf. Deshalb sollten Impflücken bei Frauen mit Kinderwunsch schon vor einer Schwangerschaft abgeklärt und geschlossen werden. Mit Totimpfstoffen, etwa gegen Pertussis, lässt sich ohne zeitliche Einschränkung vor einer Schwangerschaft impfen. Nach Impfung mit Lebendvakzinen (Masern, Mumps, Röteln, Varizellen, Gelbfieber) sollte aber vier Wochen lang verhütet werden.

Schwangere zu impfen kann manchmal nötig sein, etwa wenn ein Erregerkontakt einen postexpositionellen Schutz erfordert. Außer der Impfung gegen Influenza hält Knuf die Impfungen gegen Hepatitis A, Polio und Pneumokokken nach sorgfältiger Abwägung bei

Vorbehalte gegen Impfungen

\section{Ärzte können am besten} vom Nutzen überzeugen

- Impfungen lassen sich nur durchsetzen, wenn die Bevölkerung vom Nutzen überzeugt ist. Dabei gilt es mit Argumenten anzusetzen, denn gut ein Drittel der Eltern hat Vorbehalte gegen einzelne Impfungen, wie eine bundesweite Umfrage der Bundeszentrale für gesundheitliche Aufklärung (BZgA) mit über 3002 Teilnehmern ergeben hat. Die Schlüsselrolle bei der Entscheidung für eine Impfung haben immer noch die Haus- und Kinderärzte. 93\% der befragten Eltern halten ein Gespräch mit einem Arzt immer noch für die beste aller möglichen Informationsquellen dazu.

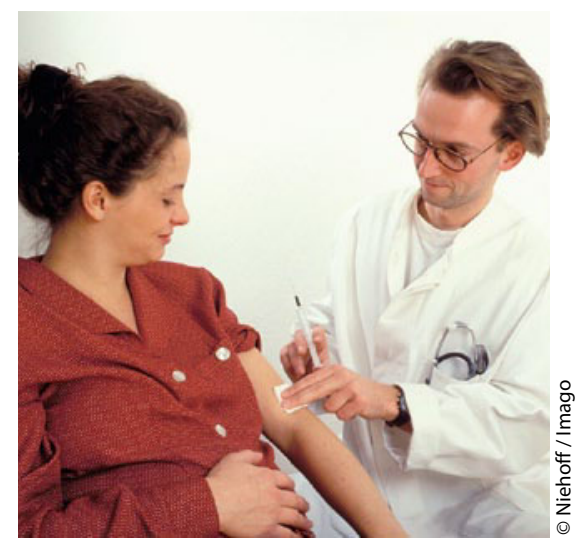

Während der Schwangerschaft sind Lebendimpfstoffe tabu.

Schwangeren für möglich. Lebendimpfstoffe sind jedoch grundsätzlich kontraindiziert. Bei Schutzbedarf sollte auf passive Immunisierungen mit Immunglobulinen ausgewichen werden.
Nachweisbarer Effekt

\section{Impfen senkt Pertussis-Prävalenz}

— „Hat ein Arzt bei Ihrem Kind jemals Keuchhusten festgestellt?" Das wurden Eltern von Viertklässlern in BadenWürttemberg zwischen 1992 und 2008 wiederholt gefragt. Außerdem wurden die Häufigkeit von Pertussisfällen in den Jahren 2007/08 mit der in den Jahren 1992/93 bis 1998/99 verglichen.

Dabei ergab sich ein deutlicher Rückgang der Lebenszeitprävalenz von Pertussis bei Viertklässlern, wie Forscher um Dr. Iris Zöllner vom Landesgesundheitsamt in Stuttgart berichten. So ging etwa in Kehl die Prävalenz von über $30 \%$ in den 1990 er-Jahren auf zuletzt $2 \%$ zurück.

Landesweit lagen die Lebenszeitprävalenzen bei den Zehnjährigen 2007/2008 im Mittel bei 10\%. Die Pertussis-Impfraten der ABC-Schützen stiegen dort gleichzeitig an: von $14 \%$ (1996) auf 92\% (2007).

Pipeline der Impfstoffhersteller ist gefüllt

\title{
Schutz gegen weitere Krankheiten in Sicht
}

- Moderne biotechnische Methoden bringen die Impfstoffentwicklung voran. Mit Spannung wird zum Beispiel ein neuer Meningokokken-B-Impfstoff erwartet, der jetzt zur Zulassung eingereicht wurde. Dieser Impfstoff könnte 2012 die letzte große Lücke beim Schutz gegen Erreger bakterieller Meningitiden schließen.

Zudem wird ein Impfstoff gegen Staphylococcus aureus in Phase II geprüft, mit dem einmal ein Großteil der jährlich 132000 Infektionen mit methicillinresistenten Staphylococcus aureus (MRSA) in Deutschland verhindert werden könnten.

Moderne Adjuvanzien haben zudem erstmals einen wirksamen Impfstoff gegen Malaria möglich gemacht. Die Vakzine verringert nach Studiendaten die
Infektionsrate und die Zahl schwerer Krankheitsverläufe deutlich und könnte nach Abschluss der Phase-III-Studien 2012 zur Zulassung eingereicht werden.

Auch neuartige therapeutische Impfstoffe gegen Krebs sind in Sicht, wobei man sich die Immunabwehr gegen spezifische Krebsantigene zunutze macht: Ein Kandidat gegen nicht kleinzelligen Lungenkrebs wird bereits in Phase-III-Studien geprüft. Nach Studiendaten kam in Folge der Impfung bei knapp einem Drittel der Patienten die Krankheit zum Stillstand oder die Tumoren bildeten sich zurück. Wenn sich das Konzept auch zukünftig bewährt, sind Impfstoffe gegen weitere Krebsformen denkbar.

WOLFGANG GEISSEL -

- 2. Nationale Impfkonferenz, Stuttgart, 8.-9. Februar 2011 